\title{
"La calidad de los aprendizajes dentro de una organización tiene relación directa con el tipo de comunicación que se puede establecer alli"
}

\author{
Por Revista dixit \\ Fotos Pablo Porciúncula
}

Conocer, analizar y mejorar los procesos de capacitación y aprendizaje organizacional constituyen pilares dentro de su obra. Ernesto Gore, Doctor por la Universidad de Buenos Aires (Argentina) y Master of Science por la Universidad de Oregon (Estados Unidos), ha logrado combinar a lo largo de su carrera la investigación y la docencia, con el ejercicio profesional en las organizaciones. Sus numerosas publicaciones académicas y de divulgación son lectura de referencia dentro de su país y en la región. Actualmente es profesor de tiempo completo en la Universidad de San Andrés (Argentina), donde cofundó junto con el profesor Guillermo Dabos, la Maestría en Estudios Organizacionales que ambos dirigen, y es profesor visitante en el Centro de Posgrados de la Universidad Católica del Uruguay. En esta entrevista, el profesor Gore focaliza en la importancia de la comunicación para los procesos de aprendizaje en las organizaciones y comparte algunas ideas que desarrolla en su libro El próximo management, recientemente publicado.
To know, analyze and improve the training and organizational learning processes are the foundations of his work. Ernesto Gore, Ph.D. by Universidad de Buenos Aires (Argentina) and Master of Science by the University of Oregon (United States), has managed to combine, during his career, research and teaching with professional work within organizations. His numerous academic publications constitute benchmark reading within his country and the region. Currently, he is a full-time professor at Universidad de San Andrés (Argentina), where he co-founded and manages, alongside professor Guillermo Dabos, the Master's Program in Organizational Studies. He is also a visiting scholar at the Postgraduate Center at Universidad Católica del Uruguay. In this interview, professor Gore focuses on the importance of communication for the learning processes in organizations and shares some ideas explored in his recently published book, El próximo management. 


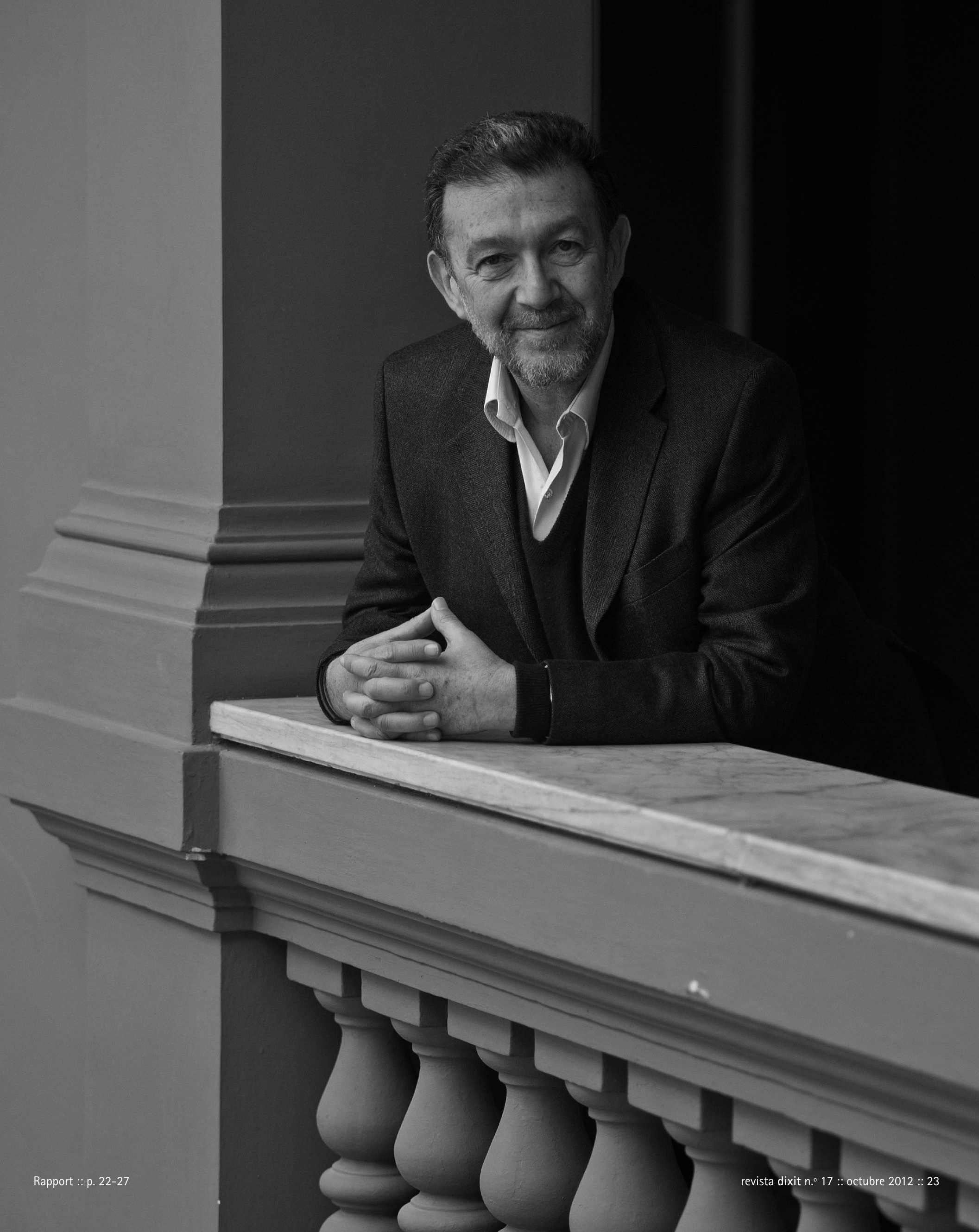




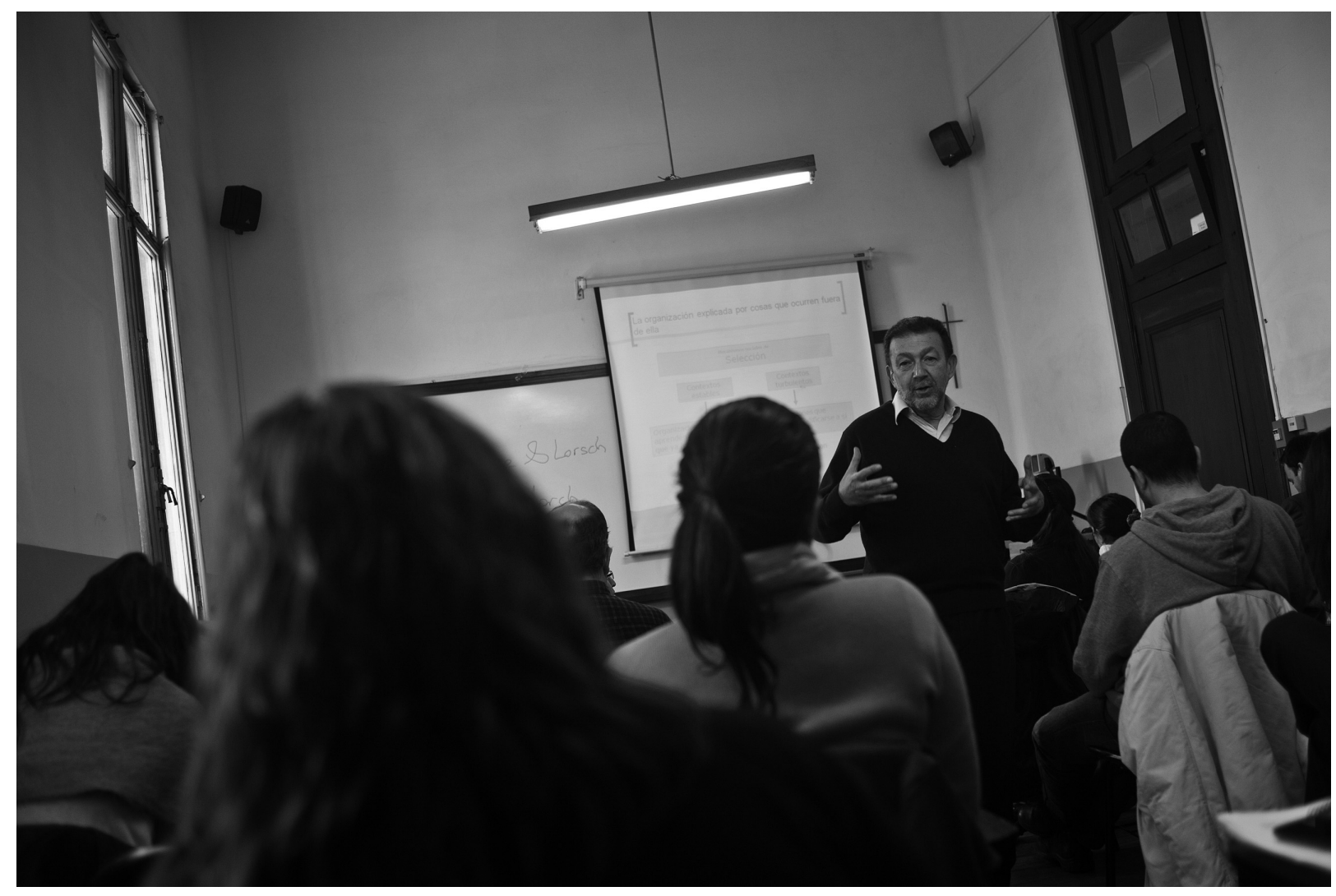

En sus trabajos sobre aprendizaje en las organizaciones, le otorga a la comunicación un rol destacado, ¿de qué manera se amalgaman estos procesos? Si estamos hablando de la relación entre comunicación y conocimiento es importante tener claro a qué nos referimos cuando hablamos de conocimiento. Al respecto hay una historia que a mí me gusta contar porque cuando la leí entendí una cantidad de cosas. Parece ser que en el Perú había una aldea donde los indigenas se parasitaban continuamente porque el río estaba parasitado. Entonces médicos y agentes sanitarios decidieron ir casa por casa, explicándoles que había que hervir el agua. Una frustración, porque tiempo después constataron que nadie lo hacía. Entonces, alguien se tomó el trabajo de reiterar la visita y les preguntó a los indígenas por qué no hervían el agua. En primer lugar respondieron que el agua hervida tenía feo gusto. Además en esa zona no había leña; conseguirla daba mucho trabajo y ellos trabajaban catorce horas diarias en la zafra. No podían después de cada jornada ir caminando a buscar leña, ni mandar a los chicos porque querían que fueran a la escuela. Y además, decían, no tenía sentido hervir el agua para después dejarla enfriar.
Entonces se entabló el siguiente diálogo:

- ¿Pero a ustedes les explicaron que en el agua hay bichos que causan las enfermedades?

-Sí, pero yo nunca los vi.

-Bueno, pero los médicos sí.

-No; yo les pregunté a los médicos y ellos tampoco los habían visto. Les habían dicho que había y además las enfermedades no las causan esos bichos que ni siquiera existen, las enfermedades se causan por el mal de ojo, problemas con los enemigos que uno tiene.

Aquí hay un problema de comunicación, pero no es un problema técnico. Los indígenas habían entendido bien la información, pero lo que estaba en juego era un sistema de creencias diferente. Comunicarse hubiera implicado no solamente trasmitir la información sino poder replantear el vínculo y el sistema de creencias. La acción humana no solamente necesita la información, también necesita de sentido, de vínculo, y de creencias.

Estas ideas se vinculan con otro de los conceptos señalados en sus trabajos: que los aprendizajes dependen de coordinaciones previas. Claro, porque 
en una organización casi todo lo que yo quiera hacer va a requerir de la ayuda de otros. En una organización puedo elegir si me voy a lavar las manos con o sin jabón, pero no muchas cosas más. Es más, puedo decidirlo siempre y cuando haya jabón disponible, cosa que depende de otros. Sin embargo, es muy frecuente pensar que si una persona adquiere determinada habilidad (que puede realizar de manera exitosa en el aula), la podrá realizar luego en el lugar de trabajo. Pero no necesariamente será así. Por un lado, porque en el trabajo tendrá otras presiones, como por ejemplo, el tiempo. Y por otro, porque para realizar las tareas en los ambientes laborales, no basta con mi conocimiento y mi intención de hacer las cosas, sino que además es necesario que otros estén convencidos y tengan la voluntad de generar las condiciones necesarias para que yo desarrolle mi tarea.

De allí el trípode que usted señala como indivisible entre la organización, el aprendizaje y la comunicación. Seguro, porque en general se piensa que la comunicación circula como por cañerías dentro de una organización. Ayuda empezar a entender un poco más claramente las cosas cuando uno se da cuenta de que la organización misma está hecha de comunicación. La organización es un sistema de acuerdos, de pedidos, es un sistema de declaraciones y de reconocimientos recíprocos, en el que la comunicación es absolutamente constitutiva. La calidad de los aprendizajes que se pueden producir allí tienen relación directa con el tipo de vínculos, o sea, con el tipo de comunicación que se puede establecer. Hay organizaciones que gastan más energía en sus guerrillas internas que en modificar el medio ambiente o en competir contra sus competidores. En esos casos hay cantidad de conocimiento, pero en tanto está segmentado, dividido, aislado y ocultado, la calidad de los aprendizajes es muy pobre. Entonces uno dice: eso que estoy describiendo ¿qué es?; ¿es un fenómeno de aprendizaje, es un fenómeno organizativo, o es un fenómeno comunicacional? Yo digo que es el mismo fenómeno de calidad de vínculos, que tiene manifestaciones en aprendizaje, en comunicación y en organización.

Usted vincula además la capacidad de aprender con la posibilidad de cuestionar los temas que a priori son incuestionables. En todo grupo humano hay temas de los que no se puede hablar, eso que Chris Argyris llamaba "los indiscutibles". Estos en algún momento pueden ser restricciones que habilitan aprendizajes, porque es necesario mantener ciertos elementos estables. Pero en determinadas circunstancias pueden convertirse en trabas terribles. Ahí es cuando Argyris dice que el crecimiento de una organización se puede medir en función de la cantidad de cosas que eran indiscutibles, y que ahora se pueden poner en discusión. Aunque sea para mantenerlas, es importante haberlas podido discutir.

En cada caso, será clave contar con personas capaces de poner esas ideas en discusión. Justamente, se trata de los fenómenos de liderazgo. No hay que confundir autoridad con liderazgo, son dos cosas distintas. Puede haber una sin la otra. El liderazgo puede darse aun sin autoridad. Líderes suelen ser aquellos que se atreven a formular las preguntas que es indispensable hacerse pero que nadie se atreve a plantear. Hablando de personas capaces, es importante tener en cuenta que no se puede explicar las organizaciones solamente a partir de las intenciones de quienes las manejan, porque en un sistema tan complejo mucho de lo que sucede está, para bien o para mal, más allá de la voluntad de los actores. Hay muchos fenómenos que surgen como respuestas improvisadas, adaptativas, a situaciones del momento que terminan siendo exitosas y son luego adoptadas como una estrategia. Pero en realidad, si uno se detiene a observar cómo fueron construidas, se da cuenta de que estas estrategias actuales en su momento se hicieron como respuestas improvisadas (pero acertadas) a problemas reales. La 


\section{Ernesto Gore}

Es doctor por la Universidad de Buenos Aires (orientación administración); master of Science por la Universidad de Oregon (Estados Unidos) y licenciado en Ciencias de la Educación por la Universidad de Buenos Aires (Argentina).

Entre 1983 y 1999 fue profesor de grado y posgrado en la Universidad de Buenos Aires.

Es profesor asociado en la Universidad de San Andrés, en Buenos Aires, en la cual creó en 2007 con el profesor Guillermo Dabos, la Maestría en Estudios Organizacionales que actualmente ambos dirigen. Es profesor invitado en la Maestría de Estudios Organizacionales de la Universidad Católica del Uruguay. Fue profesor invitado en Harvard University y en la ESCP-Europe de París.

Tiene amplia experiencia como consultor de empresas en el área de capacitación laboral y aprendizaje organizacional.

improvisación solamente es un error cuando está en manos de improvisados. En manos de gente que sabe lo que hace, puede ser, en sí misma, una estrategia.

En su libro El nuevo management, usted profundiza en los estilos de gerenciamiento actuales. ¿Cuáles son las principales características de este modelo? Una característica es que en la actualidad el rol de la dirección no tiene tanto que ver con decirle a la gente qué es lo que tiene que hacer, sino con ayudarlos a hacer lo que ellos mismos creen que tienen que hacer. Eso muchas veces implica ayudarlos a entender la organización y su contexto, pero lo que van a tener que hacer lo deberán decidir ellos mismos. Esto no siempre fue así. Hubo un tiempo en que el management consistía en decirle a la gente qué era lo que tenía que hacer, cuándo y cómo hacerlo. Hoy en día eso es cada vez más difícil. En primer lugar, porque cada vez más suceden cosas que no entendemos bien. En segundo lugar, porque es común que tengamos a nuestro cargo gente que sabe más que nosotros, sobre todo en las áreas específicas que ellos mismos manejan. Esta visión del management implica una pérdida de control, situación que genera nerviosismo en cualquier gerente. Porque su función está asociada a planificar, organizar y dirigir. Y porque las organizaciones están pensadas desde ese lugar: son sistemas de control y de autoridad. Los ferrocarriles, la línea de montaje, casi todas las cosas que hacen al mundo moderno y al
Entre sus principales publicaciones se destacan:

El próximo management: acción, práctica y aprendizaje. Editorial Granica, 2012.

Hacer visible lo invisible. Una Introducción a la formación en el trabajo, en colaboración con M. Vázquez Mazzini, Editorial Granica, Buenos Aires, 2010.

Conocimiento Colectivo, Editorial Granica, Buenos Aires, 2006.

La Educación en la Empresa. Aprendiendo en contextos organizativos, Editorial Granica, Buenos Aires, 1996.

Aprendizaje y Organización. Una lectura educativa de las teorías de la organización, en colaboración con Diane Dunlap, Buenos Aires, Editorial Tesis, 1988.

gore@udesa.edu.ar

mundo civilizado no se hubieran hecho sin los sistemas de autoridad y de control que tenemos. El solo hecho de pensar que eso pueda perder vigencia crea desasosiego. Sin embargo, pensemos en Internet, ¿dónde están los headquarters, ${ }^{2}$ la casa central de Internet? ¿Quién es el gerente general de Internet? ¿Quién es el gerente financiero de Internet? Internet no tiene nada de eso; es una organización que es una red de redes, que funciona en base a sistemas de acuerdos. ¿Quién manda allí? ¿Cuál es su objetivo? ¿Cuál es su plan estratégico? Están apareciendo formas organizativas nuevas. Probablemente nuestros modelos mentales estén demasiado atados al siglo XIX, cuando estamos en el siglo XXI. No estoy diciendo dejar de controlar, sino permitirse la perplejidad y la duda.

¿De qué manera se logran articular ambos estilos de gerenciamiento? Cada vez es más común que las áreas de ajuste rígido convivan con las áreas de ajuste flojo. Tiene que haber espacios de libertad para poder enfrentar lo que no se conoce, hacer lo que no se sabe e innovar. Por ejemplo, una empresa que tenga altos estándares de innovación, como Apple, por supuesto que tiene que ser eficiente. Pero si su único objetivo fuera la eficiencia seguiría fabricando la misma Apple que fabricaba en el año 1981, con lo cual estaría fundida. Fue por buscar, innovando en lo que no sabian hacer (lo que no es precisamente eficiente), que lograron crecer. Pero, si pensamos en una empresa de 


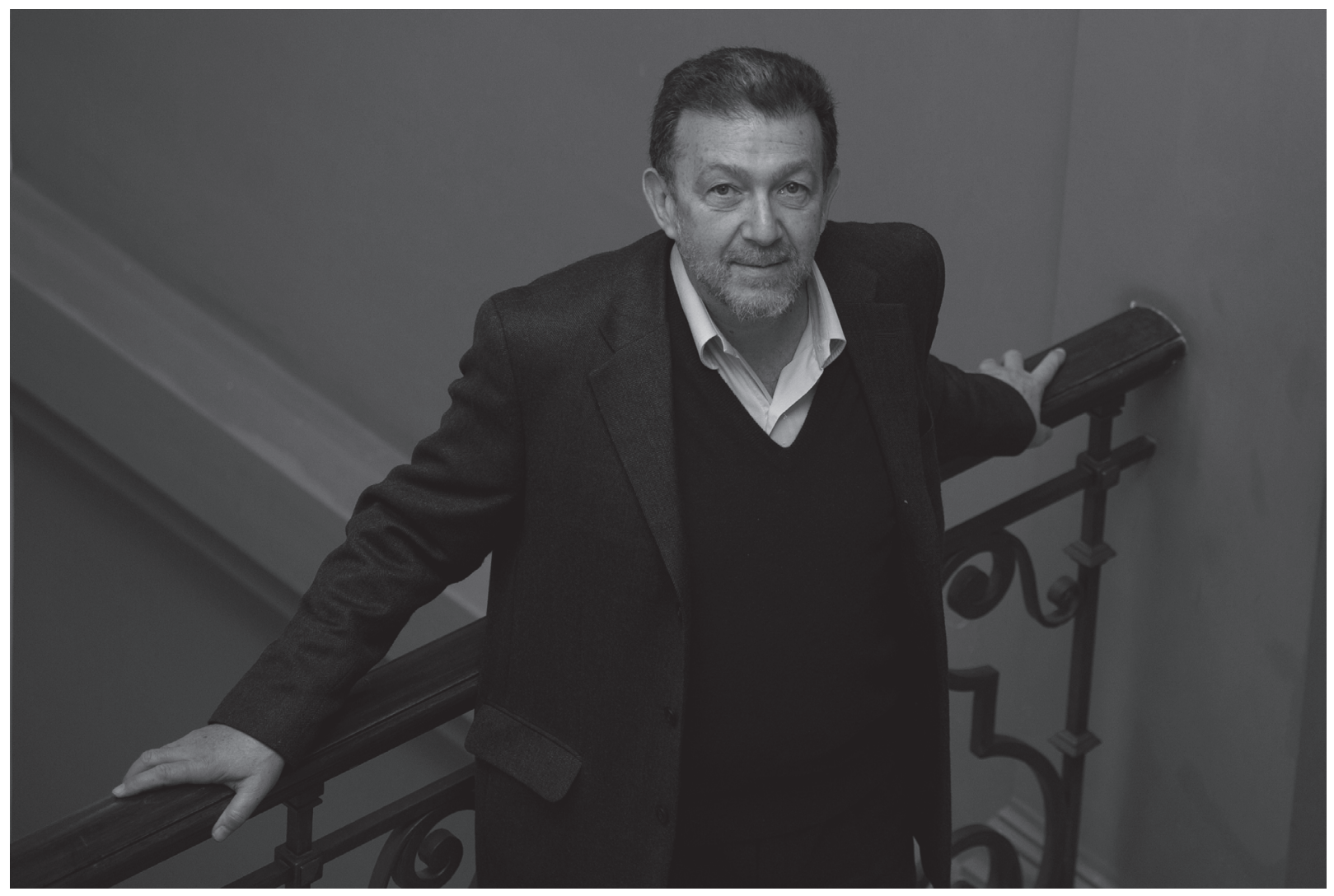

alta tecnología, en una central nuclear, su principal objetivo no es la eficiencia, sino la confiabilidad. No corresponde que tome riesgos en la seguridad para ser más eficiente, porque el costo de un error sería mucho más alto que cualquier eficiencia relativa que se pudiera lograr.

Cada vez más hace falta gente con formación generalista que pueda enfrentar cosas para las que no sabemos exactamente qué es lo que se deberá hacer, tienen que estar preparados para enfrentar lo inesperado. La formación generalista no es el conocimiento técnico específico, tampoco son puros rasgos de personalidad heredado. Es un tipo de conocimiento de base fuerte que no caduca muy fácilmente. A mí me pueden enseñar una cantidad de tecnología específica y probablemente lo más útil de ese proceso sea aprender a relacionarme con ella; porque yo sé que esos saberes van a ser viejos dentro de cinco años y voy a tener que ser capaz de adquirir otros. Hay conocimientos que sirven para adquirir otros, los más típicos son leer y escribir. También lo son sumar y restar, hacer representaciones gráficas de situaciones complejas, buscar información cuando la necesito, poder sostener un argumento, poder entender los puntos de vista de otros.

¿Qué reflexión le merecen las organizaciones de este siglo respecto a las anteriores? Yo creo que las organizaciones nunca estuvieron en una situación terriblemente distinta a la actual. Cuando pasaron de ser artesanales y familiares, a ser conglomerados industriales con líneas productivas y sistemas de producción en masa, también hicieron una transición importante. El esfuerzo adaptativo es permanente, es constante, siempre se están enfrentando cosas que uno no sabe lo que son y que requieren respuestas nuevas. $\mathrm{Y}$ algo característico de lo humano es que como especie sobrevivimos a través de acciones colectivas; ningún humano hubiera podido capturar un mamut. Porque se pudieron juntar y actuar juntos pudimos sobrevivir como especie. Y eso sigue siendo cierto. 0 sea, el fenómeno comunicacional tiene que ver directamente con la organización, el aprendizaje y la supervivencia.:. 